\title{
Anquilosis de ATM. Riesgos Anatómicos y Potencialidad del Sistema Piezoeléctrico
}

\author{
TMJ Ankylosis. Anatomical Risk and Piezoelectric System Potentiality
}

\author{
Juan Pablo Alister*; Francisca Uribe*; Sergio Olate".**; Alejandro Unibazo* \& Andrés Almeida*
}

ALISTER, J.P.; URIBE, F.; OLATE, S.; UNIBAZO, A. \& ALMEIDA, A. Anquilosis de ATM. Riesgos anatómicos y potencialidades del sistema piezoeléctrico. Int. J. Morphol., 32(2):646-651, 2014.

RESUMEN: El objetivo de este trabajo es presentar los riesgos anatómicos en el abordaje de la anquilosis de la articulación temporo mandibular (ATM) y el uso del sistema piezoeléctrico para realizar la resección de la misma. La paciente sexo femenino, 12 años, presento una anquilosis de la ATM derecha, previamente operada en base a reconstrucción con un injerto costocondral. Luego de 4 años de realizada la primera reconstrucción se presentó con una nueva anquilosis del área presentando una masa de tejido óseo de 31 $\mathrm{mm}$ en sentido latero-medial, que abarcaba hasta el foramen oval en la base de cráneo y de $28 \mathrm{~mm}$ en sentido anteroposterior. La lesión fue abordada con un acceso preauricular y posterior resección con sistema piezoeléctrico, utilizando una técnica de resección en bloque. Se presenta la técnica y se discute la potencialidad de realizar este procedimientos con los nuevos sistemas piezoeléctrico.

PALABRAS CLAVE: Anquilosis de ATM; Sistema piezoeléctrico; Osteotomía

\section{INTRODUCCIÓN}

La cirugía con sistema piezoeléctrico ha sido documentado en otras cirugías del esqueleto facial y ha demostrado resultados controversiales (Landes et al., 2008), especialmente apuntando al mayor tiempo invertido durante la cirugía; sin embargo, nuevos sistemas han mejorado aspectos como la rapidez del corte (Olate et al., 2013) y la ergonomía del equipo (Guilles et al., 2013), lo cual trae mejores condiciones para el empleo de estos sistemas.

En cirugía de ATM, recientemente se han presentado algunas indicaciones para el empleo de este sistema (Olate et al., 2014), pero en el manejo de anquilosis de ATM han sido mínimos los reportes entregados (de Castro e Silva et al., 2011). La anquilosis de ATM es un procedimiento de alta complejidad ya que la masa de tejido ósea muchas veces presenta tamaños y proyecciones que llegan a estructuras vasculares del área mediana de la base de cráneo, lo cual hace que la osteotomía para liberación sea una de las etapas mas complejas de la cirugía (Ko et al., 2009).

El objetivo de este articulo es presentar un caso de reanquilosis de ATM con una gran masa anquilótica, cuya osteotomía fue realizada con sistema piezoeléctrico, discutiendo el abordaje terapéuticos y las potenciales complicaciones anatómicas.

\section{REPORTE DEL CASO}

La paciente, sexo femenino, de 12 años de edad se presento en la División de Cirugía Oral y Maxilofacial de la Universidad de la Frontera bajo quejas de limitación de abertura bucal. La historia previa de la paciente fue de una infección no descrita a temprana edad desarrollando una posterior anquilosis de la ATM derecha; esta fue operada con resección de la masa anquilótica y la instalación de injerto costocondral junto a un colgajo de músculo temporal. La paciente evolucionó bien en la fase inicial pero luego se agudizo el cuadro clínico volviendo a formar una anquilosis de la misma articulación.

En el examen inicial se presentaba abertura máxima de $5 \mathrm{~mm}$, con movimientos de lateralidad de 1 a $2 \mathrm{~mm}$; facialmente presentaba una asimetría evidente, disminución de la altura posterior y posición del mentón retruída con respecto a la maxila y al cráneo. El estudio con imagen tomográfica evidencio una masa anquilótica de $30 \mathrm{~mm}$ en sentido medio lateral y $31 \mathrm{~mm}$ en sentido anteroposterior; la posición mediana de la masa estaba próxima del foramen oval y redondo de la base de cráneo (Figs. 1 y 2).

Se decidió la nueva cirugía de resección de la masa anquilótica, para lo cual se utilizo la técnica con sistema piezoeléctrico (Piezotome Solo®, Satelec, Francia). En la

* División de Cirugía Oral \& Maxilofacial \& Grupo CIMA, Universidad de La Frontera, Chile.

** Centro de Investigaciones Biomédicas, Universidad Autónoma de Chile, Chile. 


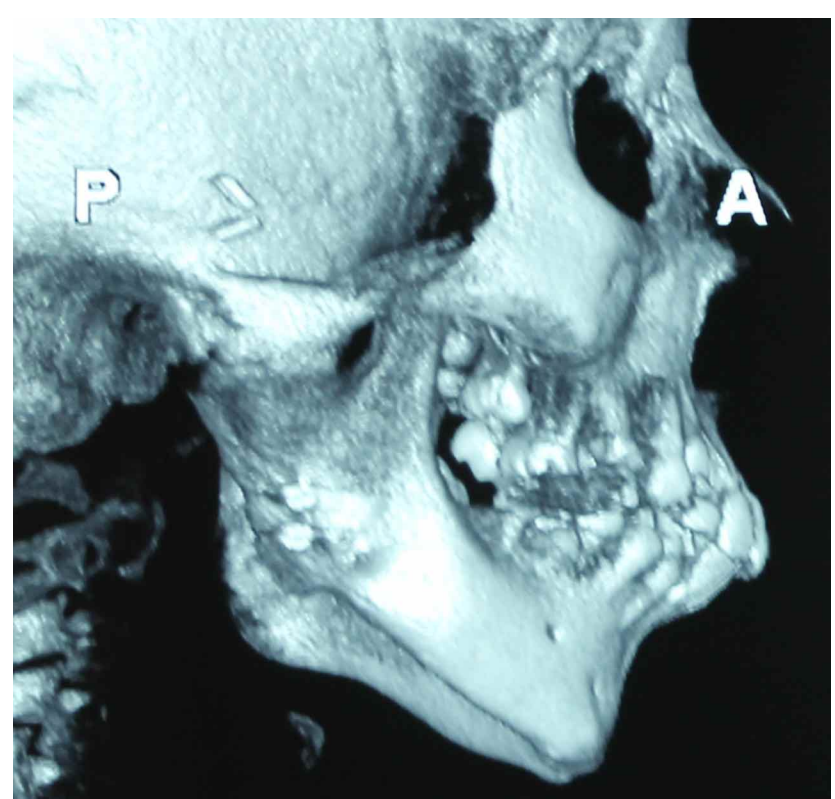

Fig. 1. Imagen en tomografía computadorizada cone beam lateral (TCCB) de ATM derecha evidenciando una masa anquilótica de gran tamaño en el sentido anteroposterior.

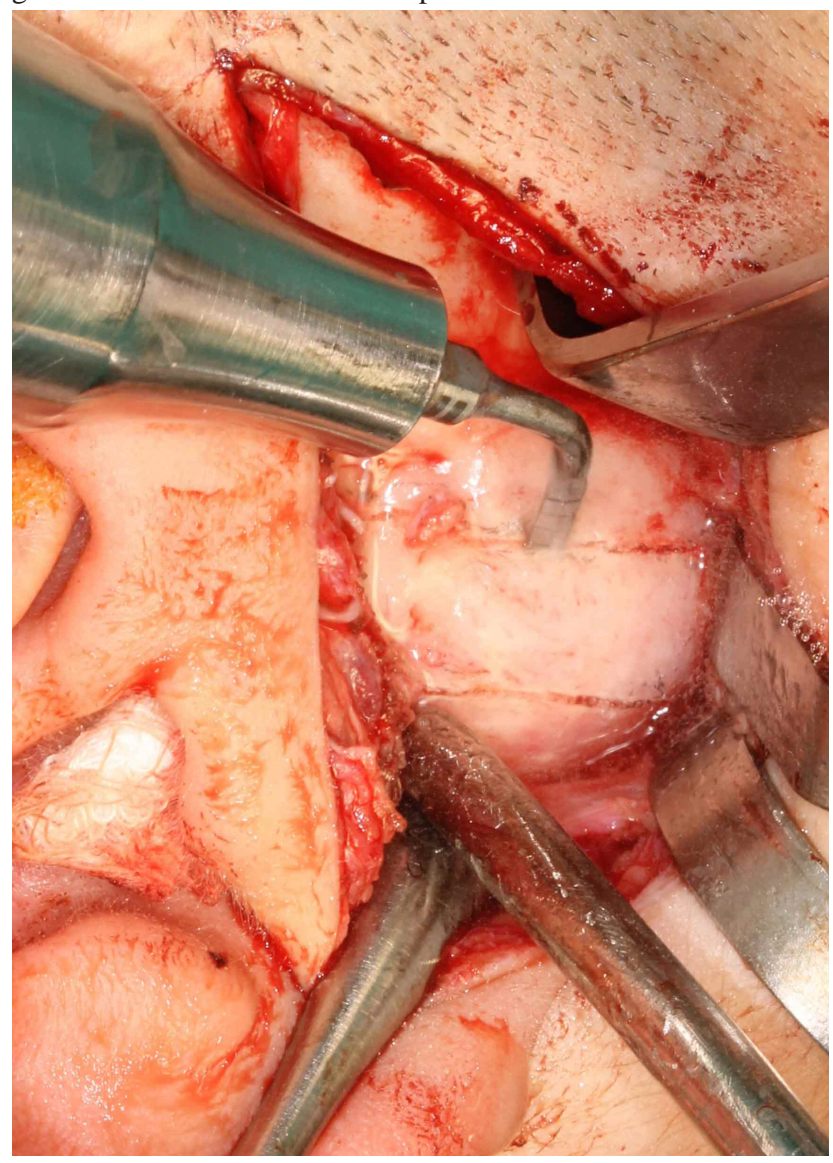

Fig. 3. Osteotomía superior realizada con el sistema ultrasónico Piezotome Solo mediante el inserto BS1; se aprecia la irrigación de suero fisiológico integrada al equipo y una separación del colgajo controlada y limitada.

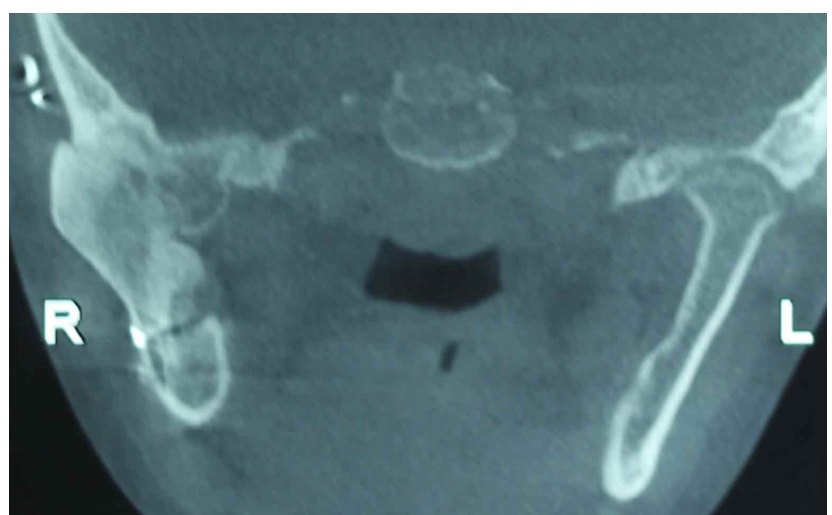

Fig. 2. Imagen de TCCB coronal que demuestra la extensión de $31 \mathrm{~mm}$ de la anquilosis de ATM derecha, con extenso compromiso de la base de cráneo.

primera etapa se realizó un abordaje con acceso preauricular, obteniendo total visibilidad de la masa anquilótica; previamente fue analizada la tomografía computadorizada cone beam (TCCB) para medir las distancias a las cuales debería llegar la osteotomía. Luego se trazó la línea del arco cigomático y se procedió a realizar la primera osteotomía lineal sobre ese sector (Fig. 3); posteriormente se midió 12 $\mathrm{mm}$ hacia inferior para realizar la segunda osteotomía lineal, ambas paralelas (Fig. 4).

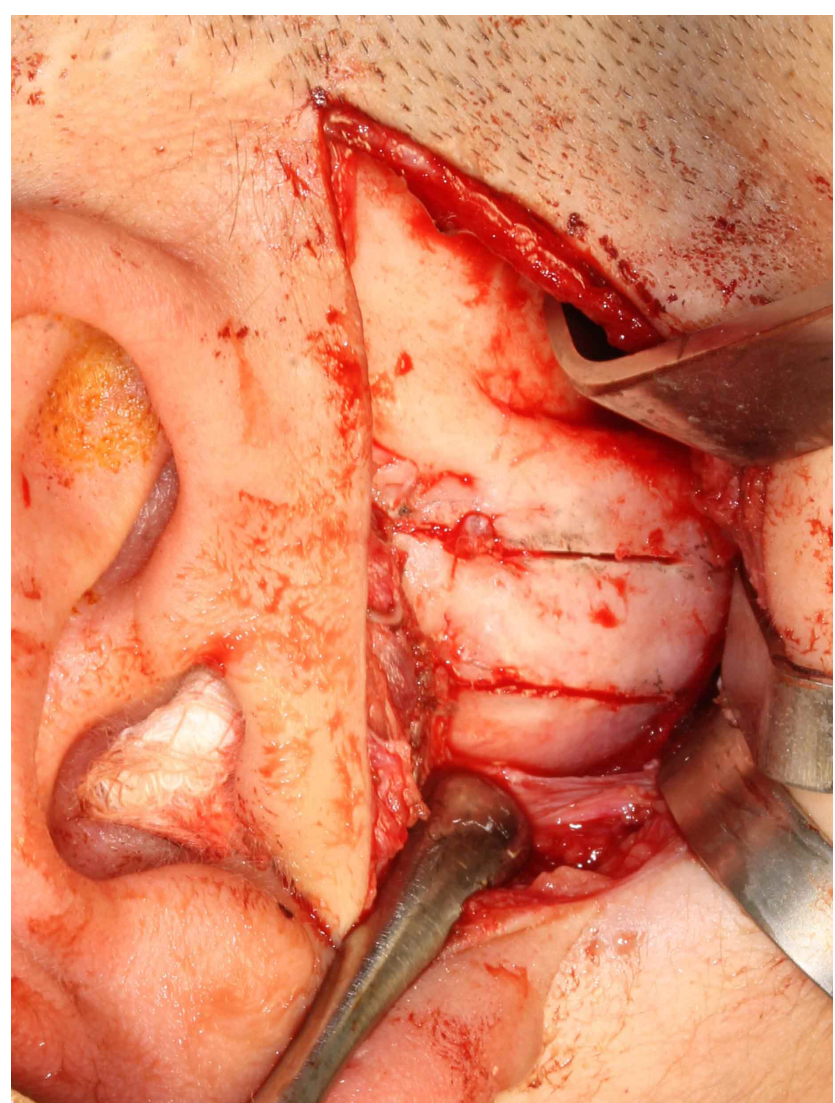

Fig. 4. Osteotomía superior e inferior realizada con las dimensiones obtenidas en el análisis de la imagen de TCCB 
La primera profundización hacia medial fue de 10 mm y se utilizo el inserto "BS1 Slim" para la maniobra; a continuación se realizó una nueva osteotomía lineal siguiendo en profundidad la primera osteotomía superior y así, secuencialmente, al igual que las otras osteotomías previamente realizadas. En esta etapa se utilizo el inserto "BS1 Long", cuyo largo presenta $15 \mathrm{~mm}$, adecuado para la técnica (Fig. 5). A continuación se procedió a realizar múltiples osteotomías verticales para retirar los fragmentos de la masa ósea (Fig. 6).

Se consiguió retirar todos los fragmentos óseos de la masa anquilótica mediante el uso de este sistema, lo cual permitió liberar la anquilosis de la ATM y generar el espacio deseado para la movilidad de la zona articular (Fig. 7). Se confirmo un bajo nivel de sangramiento, adecuada visibilidad y ausencia de lesiones en cualquier tejido blando periférico a la anquilosis.

Finalmente se procedió a la reconstrucción de la rama mandibular según la técnica descrita por Bansal et al. (2014), cuyas osteotomías para el montaje del distractor osteogénico

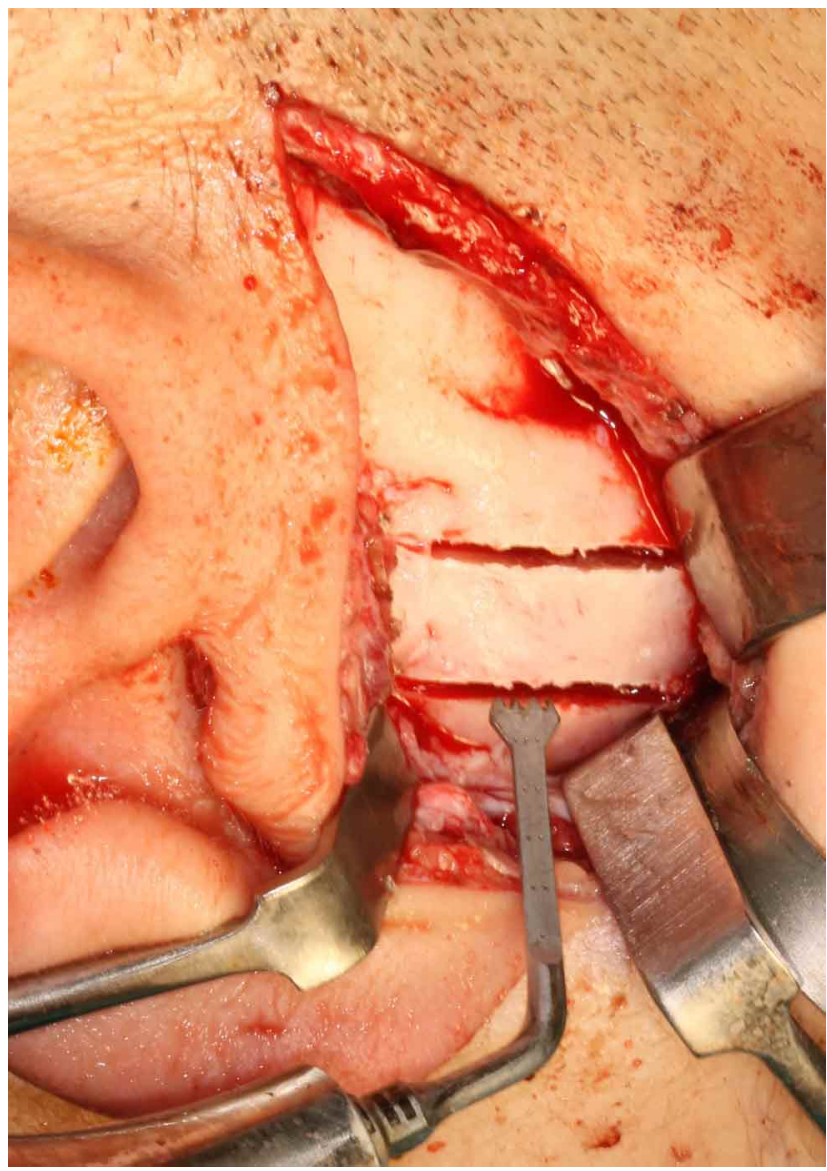

Fig. 5. Profundización de la osteotomía con el inserto "BS1 Long", que permite invadir estructura ósea con extensión de $15 \mathrm{~mm}$ de corte. interno también fueron realizadas con el sistema piezoeléctrico. La imagen de TCCB postoperatoria demuestra la resección de la masa de anquilosis de forma total, con un espacio adecuado para la fisioterapia y movilidad mandibular.

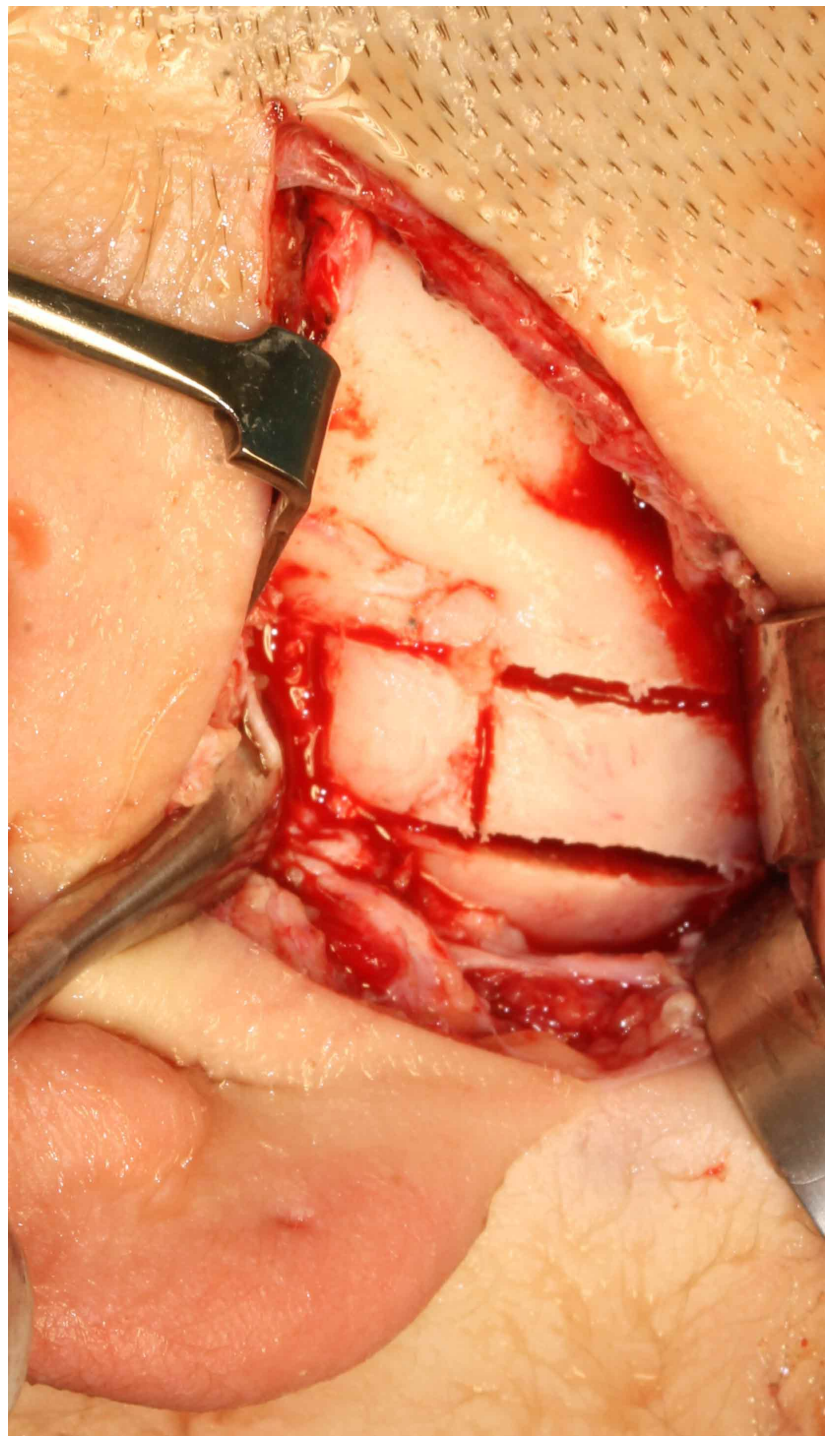

Fig. 6. Osteotomía de segmentada lateral que permite retirar cada fragmento individualmente y continuar las profundizaciones hasta obtener total liberación.

\section{DISCUSIÓN}

La artroplastia de ATM es una técnica conocida y con largos años de experiencia. Su empleo responde a variadas patologías, considerando la hiperplasia condilar, bloqueos mandibulares, trauma y también la anquilosis de ATM. 


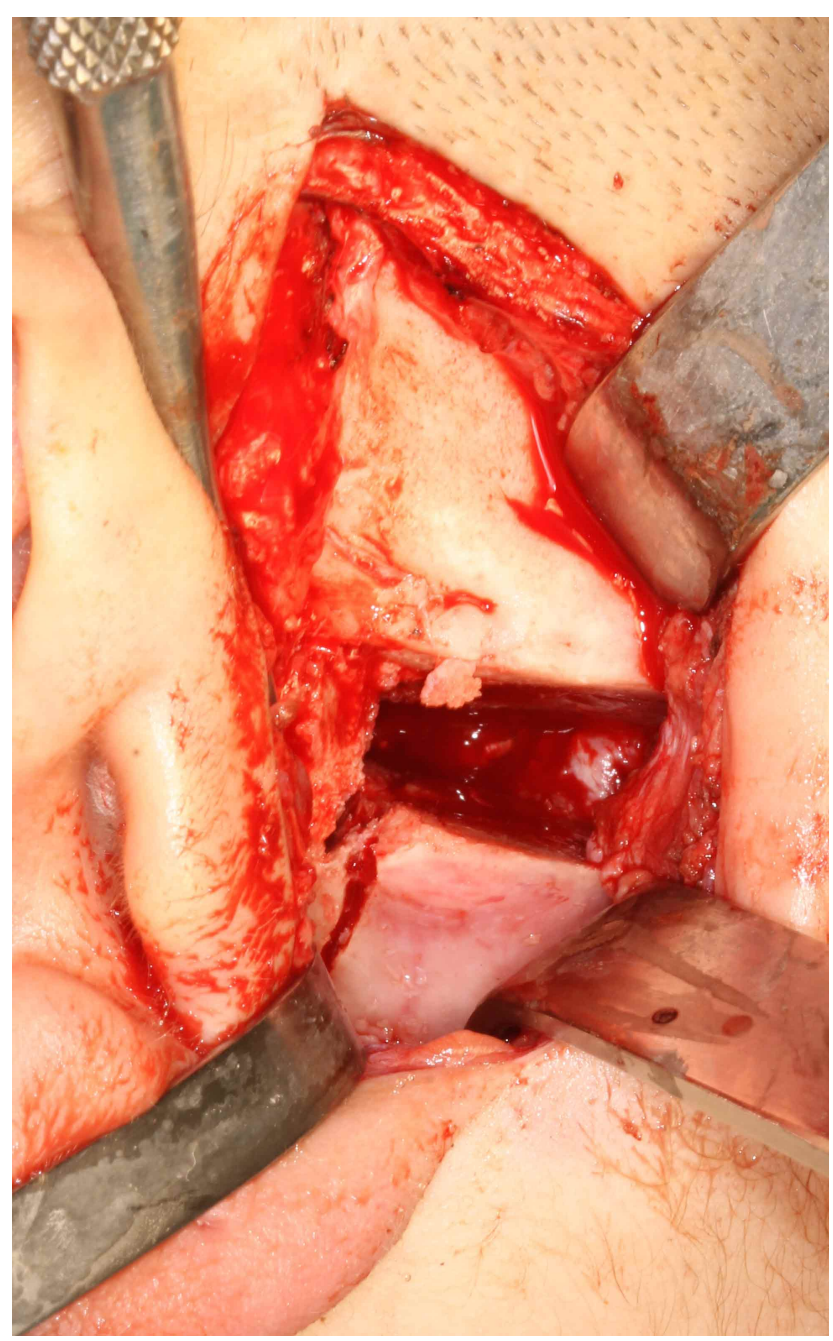

Fig. 7. Extensión de la osteotomía hasta el área medial de la masa anquilótica, demostrando limitado sangramiento y nitidez en los cortes; estructuras vasculares libres de complicaciones.

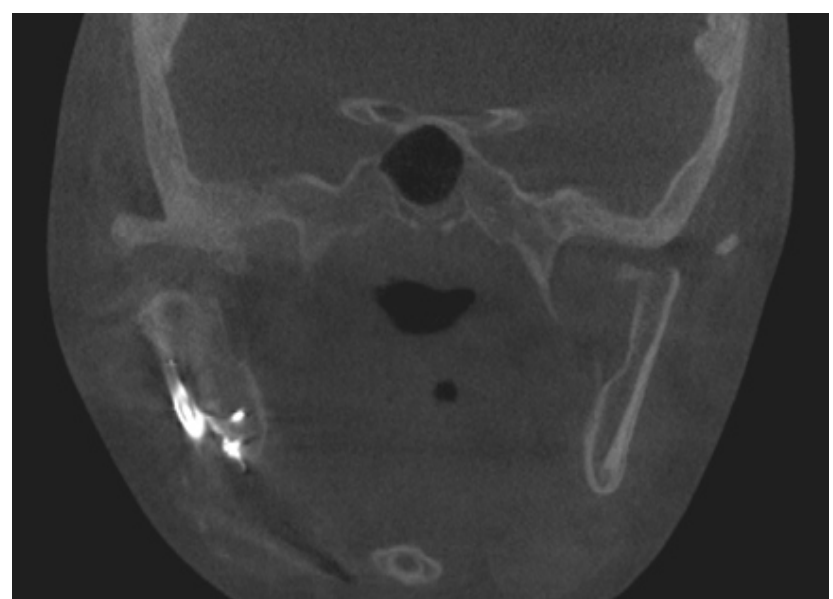

Fig. 8. TC postoperatoria mostrando una adecuada liberación de la masa anquilótica con un espacio adecuado para la movilización articular.
Las complicaciones asociadas a la artroplastia por anquilosis de ATM se reportan en fallas de los sistemas utilizados como distractores, injertos o prótesis de ATM o en complicaciones postoperatorias como infección o reanquilosis (Erol et al., 2006); las complicaciones vasculares se observan en pocas investigaciones y su manejo se incorpora como parte de la metodología de tales estudios (Elgazzar et al. 2010).

Kaban et al. (2009) señalan que parte importante del éxito del procedimiento esta en un retiro agresivo de la masa ósea/fibrosa de anquilosis, lo cual exige extensos cortes, que este sistema piezoeléctrico pueden desarrollarse con mayor seguridad apuntando a no dañar tejidos blandos vasculares o nerviosos (Labanca et al. 2008). Itro et al., 2012 presentó un caso donde se realizaba una artroplastia con sistema piezoeléctrico, señalando su eficiencia y seguridad para desarrollar el corte; sin embargo, el nivel de compromiso fue menor al presentado en este caso.

La proximidad de grandes vasos y nervios en este caso fue de una importante consideración durante la cirugía, pero gracias al uso del sistema piezoeléctrico disminuyo el potencial de daño a estos (Shirota et al., 2014). Elgazzar et al. (2010), señalaron que la osteotomía de la anquilosis era realizada con brocas y cinceles con especial cuidado en la arteria maxilar, controlando la hemorragia con paquetes de gaza; los autores del presente reporte estiman que con el uso del sistema piezoeléctrico se puede optimizar la cirugía con un bajo riesgo de daño a tejidos blandos. Por otra parte, el menor grado de sangramiento, mejor visibilidad y la irrigación constante del sistema son de gran ayuda durante cirugías exigentes como lo son estos casos de artroplastia en reanquilosis de ATM.

Anatómicamente, la ATM presenta estructuras vasculares y nerviosas conocidas. Topográficamente, las estructuras vasculares asociadas a la ATM están representadas por la arteria temporal superficial y la arteria facial transversa en el aspecto lateral del cuello condilar y también por la arteria maxilar y el plexo pterigoide en el aspecto medial (Orbay et al., 2007). La masa anquilótica impide un reconocimiento normal de las estructuras debido al crecimiento de tejido óseo y fibroso junto a la base de cráneo, de forma que otras estructuras vasculares y nerviosas pueden estar próximas al área de la masa óseo, de forma que extensas masas son capaces de comprometer forámenes y estructuras vasculares mediales como la arteria carótida interna (Eller et al., 2012).

Se ha sugerido que la embolización puede ser una alternativa para mejorar el pronóstico intraoperatorio y disminuir los riesgos de hemorragias (Susarla et al., 2014); la 
embolización implica un procedimiento con anestesia general que también implica riesgos como la migración y embolización cerebral, alergias y evidentemente aumenta los costos del procedimiento (Bouloux \& Perciaccante, 2009; Frame et al., 1987).

La osteotomía con sistema peizoeléctrico, gracias a la naturaleza de su acción mecánica y al hecho de no realizar cortes sobre tejidos blandos (arterias, músculos y nervios, entre otros) (Olate et al., 2013), podría llevar a que no sea necesario una embolización debido al bajo riesgo de lesionar estructuras vasculares. Por otra parte, este sistema de osteotomía permite que el acceso quirúrgico preauricular convencional, de menor tamaño al observado en el manejo de la artroplastia en anquilosis de ATM (Bulgannawar et al., 2011) ya que para la acción de la punta de corte se requiere una separación menos agresiva con visibilidad menos exigente (Olate et al., 2014)

Finalmente, se puede concluir que el presente caso no presentó complicaciones intraoperatorias o postoperatorias asociadas a variables anatómicas o quirúrgicas; la paciente continua en seguimiento para identificar futuros procedimientos.

ALISTER, J.P.; URIBE, F.; OLATE, S.; UNIBAZO, A. \&ALMEIDA, A. TMJ Ankylosis. Anatomical Risk and Piezoelectric System Potentiality. Int. J. Morphol., 32(2):646-651, 2014.

SUMMARY: The aim of this report is to present the anatomical risk for to treat the temporo mandibular joint (TMJ) ankylosis and the use of the piezoelectric system for to make the bone resection. Female patient, 12 years old, presented a right TMJ ankylosis, that was previously operated with a costocondral graft reconstruction. After 4 year from these reconstructive surgery the patient was involved in a new TMJ anklylosis of the same side showing a bone mass with $31 \mathrm{~mm}$ in an meddle-lateral direction, with compromise until to oval foramen in the skull base and $28 \mathrm{~mm}$ in the anterior-posterior direction. The lesion was operated by a preauricular approach and then a bone resection with the piezoelectric system, using a block resection technique. In this report it's present the technique and is discusses the potentiality of the new piezoelectric system for these procedures.

KEY WORDS: TMJ ankylosis, piezoelectric system, osteotomy.

\section{REFERENCIAS}

Bansal, V.; Singh, S.; Garg, N. \& Dubey, P. Transport distraction osteogenesis as a method of reconstruction of the temporomandibular joint following gap arthroplasty for posttraumatic ankylosis in children: a clinical and radiological prospective assessment of outcome. Int. J. Oral Maxillofac. Surg., 43:227-36, 2014.

Bouloux, G. F. \& Perciaccante, V.J. Masive hemorrhage during oral and maxilofacial surgery: ligation of the external carotid artery or embolization?. J. Oral Maxillofac. Surg., 67:1547$51,2009$.

Bulgannawar, B. A.; Rai, B. D.; Nair, M. A. \& Kalola, R. Use of temporalis fascia as an interpositional arthroplasti in temporomandibular joint ankylosis: analysis of 8 cases. J. Oral Maxillofac. Surg., 69:1031-5, 2011.

De Castro e Silva, L. M.; Pereira Filho, V. A.; Vieira, E. H. \& Gabrielli, M.F. Tracheostomy-dependent child with temporomandibular ankylosis and severe micrognathia treated by piezosurgery and distraction osteogenesis: case report. $\mathrm{Br}$. J. Oral Maxillofac. Surg., 49:e47-9, 2011.

Eller, J. L.; Sasaki-Adams, D.; Sweeney, J. M. \& Abdulrauf, S.I. Localization of the internal maxillary artery for extracranial-tointracranial bypass through the middle cranial fossa: a cadaveric study. J. Neurol. Surg. B. Skull. Base, 73:48-53, 2012.
Elgazzar, R. F.; Abdelhady, A. I.; Saad, K. A.; Elshaal, M. A.; Hussain, M. M.; Abdelal, S. E. \& Sadakah, A. A. Treatment modalities of TMJ ankylosis: experience in Delta Nile, Egypt. Int. J. Oral Maxillofac. Surg. 39:333-42, 2010.

Erol, B.; Tanrikulu, R. \& Görgün, B. A clinical study on ankylosis of the temporomandibular joint. J. Craniomaxillofac. Surg., 34:100-6, 2006.

Frame, J. W.; Putman, G.; Wake, M.J. \& Rolfe, E.B. Therapeutic arterial embolisation of vascular lesions in the maxilofacial región. Br. J. Oral Maxillofac. Surg.; 25:181-94, 1987.

Gilles, R.; Couvreur, T. \& Dammous, S. Ultrasonic orthognathic surgery: enhacements to established osteotomies. Int. J. Oral Maxillofac. Surg, 42:981-7, 2013.

Itro, A.; Lupo, G.; Carotenuto, A.; Filipi, M.; Cocozza, E.; Fiengo, G. \& Marra, A. Management of temporomandibular joint ankylosis: a case report of joint replacement with piezoelectric surgery. Minerva Stomatol., 61(7-8):329-35. 2012.

Ko, E. C.; Chen, M. Y.; Hsu, M.; Huang, E. \& Lai, S. Intraoral approach for arthroplasty for correction of TMJ ankylosis. Int. J. Oral Maxillofac. Surg., 38:1256-62, 2009. 
Landes, C.; Stübinger, S.; Rieger, J.; Willinger, B.; Ha, T. K. L. \& Sader, R. Critical evaluation of piezoelectric osteotomy in orthognathic surgery: operative technique, blood loss, time requirement, nerve and vessel integrity. J. Oral Maxillofac. Surg, 66:657-74, 2008.

Labanca, M.; Azzola, F.; Vinci, R. \& Rodella, L. Piezoelectric surgery: twenty years of use. Br. J. Oral Maxillofac. Surg., 46:265-9, 2008

Olate, S.; Almeida, A.; Unibazo, A.; Alister, J.P.; Uribe, F.; Martínez, F. \& Huentequeo-Molina, C. Osteotomías craneomaxilo-faciales con sistemas ultrasónicos. Rev. Chil. Cir., 65:454-62, 2013

Olate, S.; Unibazo, A.; Almeida, A. \& de Moraes, M. Mandibular condylectomy revisited: technical notes concerning the use of an ultrasonic system. J. Oral Maxillofac. Surg., 72:481-4, 2014.

Orbay, H.; Kerem, M.; Unlü, R.E.; Cömert, A.; Tüccar, E. \& Sensöz, O. Maxillary artery: anatomical landmarks and relationship with the mandibular subcondyle. Plast. Reconstr. Surg., 120:1865-70, 2007.

Susarla, S.; Peacock, Z.S.; Williams, W.B.; Rabinov, J.D.; Keith, D.A. \& Kaban, L.B. Role of computed tomographic angiography in treatment of patients with temporomandibular joint ankylosis. J. Oral Maxillofac. Surg., 72:267-76, 2014.

Shirota, T.; Kamatani, T.; Yamaguchi, T.; Ogura, H.; Maki, K. \& Shintani, S. Effectiveness of piezoelectric surgery in reducing surgical complications after bilateral sagital Split osteotomy. Br. J. Oral Maxillofac. Surg., 52:219-22, 2014.

\section{Correspondencia a:}

Prof. Dr. Sergio Olate, PhD

Claro Solar 115, oficina 414-A

Temuco

CHILE

Email: sergio.olate@ufrontera.cl

Recibido: 28-11-2013

Aceptado: 17-03-2014 